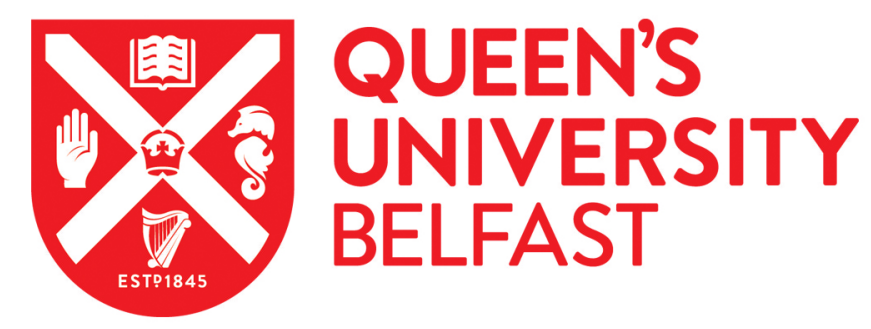

\title{
Testing Wavefunction Collapse Models using Parametric Heating of a Trapped Nanosphere
}

Goldwater, D., Paternostro, M., \& Barker, P. F. (2016). Testing Wavefunction Collapse Models using Parametric Heating of a Trapped Nanosphere. Physical Review A (Atomic, Molecular, and Optical Physics), 94(1). https://doi.org/10.1103/PhysRevA.94.010104

Published in:

Physical Review A (Atomic, Molecular, and Optical Physics)

Document Version:

Peer reviewed version

Queen's University Belfast - Research Portal:

Link to publication record in Queen's University Belfast Research Portal

Publisher rights

Copyright 2016 American Physical Society.

This work is made available online in accordance with the publisher's policies. Please refer to any applicable terms of use of the publisher.

\section{General rights}

Copyright for the publications made accessible via the Queen's University Belfast Research Portal is retained by the author(s) and / or other copyright owners and it is a condition of accessing these publications that users recognise and abide by the legal requirements associated with these rights.

Take down policy

The Research Portal is Queen's institutional repository that provides access to Queen's research output. Every effort has been made to ensure that content in the Research Portal does not infringe any person's rights, or applicable UK laws. If you discover content in the Research Portal that you believe breaches copyright or violates any law, please contact openaccess@qub.ac.uk. 


\title{
Testing Wavefunction Collapse Models using Parametric Heating of a Trapped Nanosphere
}

\author{
Daniel Goldwater, ${ }^{1, *}$ Mauro Paternostro, ${ }^{2}$ and P. F. Barker ${ }^{1}$ \\ ${ }^{1}$ Department of Physics and Astronomy, University College London, Gower Street, London WC1E 6BT, United Kingdom \\ ${ }^{2}$ Centre for Theoretical Atomic, Molecular, and Optical Physics, \\ School of Mathematics and Physics, Queen's University, Belfast BT7 1NN, United Kingdom
}

(Dated: June 23, 2016)

\begin{abstract}
We propose a mechanism for testing the theory of collapse models such as continuous spontaneous localization (CSL) by examining the parametric heating rate of a trapped nanosphere. The random localizations of the center-of-mass for a given particle predicted by the CSL model can be understood as a stochastic force embodying a source of heating for the nanosphere. We show that by utilising a Paul trap to levitate the particle and optical cooling, it is possible to reduce environmental decoherence to such a level that CSL dominates the dynamics and contributes the main source of heating. We show that this approach allows measurements to be made on the timescale of seconds, and that the free parameter $\lambda_{\text {csl }}$ which characterises the model ought to be testable to values as low as $10^{-12} \mathrm{~Hz}$.
\end{abstract}

Dynamical reduction models - better known as collapse theories - seek to resolve the measurement problem by inserting a non-linear stochastic term in the Schrödinger equation (SE). This would account for genuine collapses of superposition states. In these theories, 'localisation' events occur at a frequency scaling with the mass of the system at hand. These are fundamentally different to environmental decoherence [1], and are invoked as the origin of wavefunction collapse. Such modifications to the SE aim to provide a theory capable of describing phenomena at all scales, and are designed to reproduce conventional quantum mechanics (CQM) when dealing with small masses, and classical mechanics at the macroscopic scale.

One of the most celebrated models of dynamical reduction is the Continuous Spontaneous Localization (CSL) model [2]. It is characterised by two parameters: a length $r_{c}$, and a frequency $\lambda_{\mathrm{csl}}$. The former provides a length scale above which reduction effects would be relevant, the latter embodies the rate at which spatial superpositions of a single nucleon separated by a distance greater than $r_{c}$ would collapse. While $r_{c}$ is generally taken to be $\approx 100 \mathrm{~nm}$, the value of $\lambda_{\mathrm{csl}}$ is the subject of uncertainties [3-5] and is currently taken to span a range from $10^{-16} \mathrm{~Hz}$ [2] to $10^{-5} \mathrm{~Hz}$ [6]. A value of $10^{-8 \pm 2} \mathrm{~Hz}$ has been proposed [7], based on the process of image formation on photographic film. The heating rate of ultracold atoms was used to set a value of $10^{-7}$ $\mathrm{Hz}$ [8], while the maximum allowable heating rate of the intergalactic medium seems to be compatible with $10^{-10 \pm 2} \mathrm{~Hz}[9]$.

The effects of localization are mathematically very similar to those of decoherence [10], meaning that any experiment built to search for a signature of such a collapse mechanism must minimize the effects of decoherence as much as possible so as to better distinguish the hallmark of the former from that of the latter. However, for a given object, both its rate of localization and decoherence will increase proportionally to mass. To test collapse we must study objects large enough to have an appreciable localization rate, yet small enough that decoherence does not dominate the dynamics. The scale at which this becomes possible is the so-called mesoscopic one, the liminal scale between the well established quantum and classical regimes. Recently, Nimmrichter et al. have shown [11] that beyond a certain size collapse effects have a sub-linear scaling with size, a result which we corroborate here, and which clearly identifies the regime of interest.

Lately there have been a considerable number of proposals to test collapse theories, and the challenge of reducing decoherence manifests itself differently depending on the scheme [12-15]. Unfortunately many proposals do not include detailed analyses of conventional environmental noise, and as such, it is not clear what range of values of $\lambda_{\text {csl }}$ they could probe. Matter-wave interferometric methods are an attractive means for such tests with a well established range of testable values of $\lambda_{\text {csl }}[16]$. Such settings, however, require the preparation and detection of many identical particles, which makes implementation challenging. Optomechanical proposals [19] are attractive in comparison, because they in principle require only a line shape measurement of the light scattered by an intra-cavity macroscopic oscillator, and do not require ground state cooling. However, as such line shape would be narrow (order of $\mu \mathrm{Hz}$ ) very long times (in excess of months) would be required for the measurement. A recent proposal has been made based on dynamical decoupling [20], which is promising but may also be constrained by long testing times.

The concept of utilising the energy gain of a harmonic oscillator to test CSL was first suggested by Adler [21] and more recently re-examined in Refs. [22-24]. Whilst this method is relatively straightforward and therefore attractive for implementation, the ability to test for CSL is very dependent on a detailed and realistic inclusion of conventional decoherence mechanisms. In order for the classical approach put forward, for instance, in Ref. [24] to be effective, the collapse mechanism must be able to induce the excitation of a rather substantial number of 


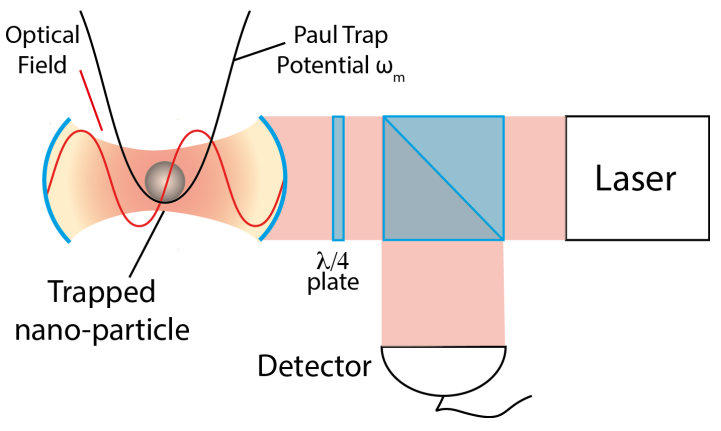

FIG. 1. Schematic of the experiment, in which the particle is levitated by the electric field of the Paul trap, and cooled by the optical cavity.

thermal phonons in a given oscillator. This is, in general, not the case for a large range of values of the parameters that characterize a given collapse model and such approaches can only assess the largest of their conjectured values.

In this paper, we propose an experimentally viable way to explore CSL on the mesoscale by utilising a cavity-cooled, single-charged nanosphere trapped in a Paul trap [26]. Measurements can be made with a single trapped particle in less than 100 seconds and, under optimal conditions, we find this scheme capable of probing $\lambda_{\text {csl }}$ to values as low as $10^{-12} \mathrm{~Hz}$, thus going significantly beyond the literature reported so far. Most importantly, our protocol allows for the discrimination between collapse effects and mis-characterised conventional noise ones- a challenge which has to our knowledge remained unaddressed so far.

We explore the possibility for an optomechanical test of a form of CSL described in Ref. [10, 11, 19], in which the effects of spontaneous localization are modelled as a delta-correlated stochastic noise source $w_{t}$. This approach is valid when the scale of spatial-superposition separations is less than $r_{c}$. The noise term $w_{t}$ will occur in the dynamical equations of the system as an extra Langevin force $[19,25]$. Its effect on the dynamics of a mechanical oscillator would depend on the size and density of the object collapsing, and the two parameters $r_{\mathrm{c}}$ and $\lambda_{\mathrm{csl}}$ characterizing the model. Conveniently, we can represent the effects of the localization process via a diffusion operator characterised by the coefficient $D_{\text {csl }}$ and appearing in the master equation describing the particle in the same way as conventional heating sources. The diffusion coefficient takes the form [11]

$$
D_{\mathrm{csl}}=\frac{\hbar}{m \omega_{m}} \frac{\lambda_{\mathrm{csl}}}{r_{c}^{2}} \alpha
$$

where $\alpha$ is a geometry-dependent factor, which for a sphere is given by

$$
\alpha=\left(\frac{m}{m_{0}}\right)^{2}\left[e^{-R^{2} / r_{c}^{2}}-1+\frac{R^{2}}{2 r_{c}^{2}}\left(e^{-R^{2} / r_{c}^{2}}+1\right)\right] \frac{6 r_{c}^{6}}{R^{6}} .
$$

Here $m_{0}=1$ amu and $R$ is the sphere's radius.

The Protocol.- We now describe the scheme that we propose to test the CSL model. We levitate a charged nanosphere in a hybrid trap consisting of a Paul trap and an optical cavity, and use them to cool its motion to a temperature corresponding to a low occupation number. We then turn off the optical field (and hence the cooling) and let the dynamics evolve for a certain amount of time before measuring the energy of the oscillator again. A model including the effects of CSL predicts it will have heated more than one would expect due to conventional noise sources alone. If the measured energy matches that predicted by conventional noise sources, we will have provided evidence against CSL to within a certain range of $\lambda_{\text {csl }}$, whereas a higher measured energy would indicate some other dynamics at play, in favour of collapse theories.

We divide the procedure into two phases: a cooling phase, and free evolution. For the purpose of testing CSL, it is the second phase that is important. In this period of free evolution the nanosphere is levitated using a single electric potential, which could be provided by a number of generic trap architectures. The mechanism of cavity cooling for nanoscale objects is well established [13, 26-37], and relies on having the particle sit in two potential wells: one of which traps, and one (or both) of which cool. Though these potentials are traditionally provided by an optical cavity populated with two distinct optical fields, Ref. [18, 26] shows that cooling is possible using a Paul trap in conjunction with a single-mode optical cavity. While both these systems are required for the cooling phase, the Paul trap alone suffices to levitate it.

This is appealing, because in the low pressure scenario of a particle levitated solely by optical fields the dominant source of heating is the scattering of cavity photons [32]. By using a hybrid trap we can cool the particle to a desired temperature and then turn off the optical field completely, leaving the particle suspended in the Paul trap alone. This ability to turn off the optical field without 'losing' the particle means that we can do away with what would otherwise be the dominant cause of heating - optical scattering - and thus greatly reduce the conventional heating sources that would otherwise mask the CSL effects.

We emphasize that although we require cooling, we do not need to achieve the ground-state energy. Indeed, the simple comparison between the initial phonon number $n_{0}$ and the final one $n_{f}$ after the period of free evolution will give us information on the heating rate [17].

The period of free evolution is governed by the Hamiltonian $\hat{H}=\hat{H}_{0}+\hat{H}^{\prime}$, where $\hat{H}_{0}=\hbar \omega_{m} \hat{a}^{\dagger} \hat{a}, \omega_{m}$ is the secular frequency of the Paul trap, $\hat{a}^{\dagger}, \hat{a}$ are the creation and annihilation operators for the centre of mass motion of the sphere respectively, and $H^{\prime}$ represents the interaction between system and environment. We can 
then solve the master equation $\dot{\rho}=-(i / \hbar)[\hat{H}, \rho]$ for the oscillator [41]. The forms of coupling to noise sources in $H^{\prime}$ determine their effects on the master equation [35]. We have explored each noise source in detail, examining collisions with the background gas, blackbody radiation, acoustic noise affecting the trap, Johnson and patch potential noise from the electrodes, micromotion from the trap's driving frequency, and anisotropy of the sphere [17]. We group these noise sources as momentum diffusion, occurring at rate $D_{\mathrm{p}}$, position diffusion at rate $D_{\mathrm{q}}$, and momentum dissipation at rate $\Gamma$, thus getting the dynamical equation [41]

$$
\dot{\rho}=-\frac{i}{\hbar}\left[\hat{H}_{0}, \rho\right]-\sum_{j=1}^{2} D_{j}\left[\hat{X}_{j},\left[\hat{X}_{j}, \rho\right]\right]-\Gamma\left[Q_{z},\left\{\hat{P}_{z}, \rho\right\}\right]
$$

where $\hat{\boldsymbol{X}}=\left(\hat{Q}_{z}, \hat{P}_{z}\right)$ is the vector of quadratures of the nanosphere $\hat{Q}_{z}=\hat{a}+\hat{a}^{\dagger}$ and $\hat{P}_{z}=i\left(\hat{a}^{\dagger}-\hat{a}\right)$, and $\boldsymbol{D}=\left(D_{\mathrm{p}}, D_{\mathrm{q}}\right)$. Our analysis of the various noise sources, including the possible effects of anisotropy of the nanosphere (and the consequent non-uniform distribution of the charge) finds all but gas collision and blackbody radiation to be negligible [17], giving us $\Gamma=$ $\left(\gamma_{\mathrm{gas}}+\gamma_{\mathrm{bb}, \mathrm{e}}+\gamma_{\mathrm{bb}, \mathrm{a}}\right) / 4$ and $D_{\mathrm{p}}=D_{\text {gas }}+D_{\mathrm{bb}}+D_{\mathrm{csl}}$, where $D_{\text {csl }}$ is given in Eq. (1) and

$$
D_{\text {gas }}=\frac{\gamma_{g} k_{B} T_{\text {env }}}{2 \hbar \omega_{m}}, \quad D_{\mathrm{bb}}=\frac{k_{B}\left(\gamma_{\mathrm{bb}, \mathrm{e}} T_{\mathrm{int}}+\gamma_{\mathrm{bb}, \mathrm{a}} T_{\mathrm{env}}\right)}{2 \hbar \omega_{m}} .
$$

Here, $\gamma_{\mathrm{gas}}, \gamma_{\mathrm{bb}, \mathrm{e}}, \gamma_{\mathrm{bb}, \mathrm{a}}$ are the damping constants related to gas collisions, blackbody emission and blackbody absorption respectively. The environmental temperature is $T_{\text {env }}, \omega_{m}$ is the mechanical frequency of the nanosphere, and $m$ is its mass.

Heating Rate of a Trapped Particle.- The mean occupation number (phonon number) of the trapped particle as a function of time $\langle n\rangle_{t}$ is determined via Eq. (2). Assuming a thermal state $[27,35,39]$, the expression for the rate of change of the average phonon number simplifies to $\langle\dot{n}(t)\rangle=-\Gamma\langle n(t)\rangle+D_{\mathrm{p}}$, which has the solution

$$
\langle n(t)\rangle=e^{-\Gamma t}\left(n_{0}-\frac{D_{\mathrm{p}}}{\Gamma}\right)+\frac{D_{\mathrm{p}}}{\Gamma},
$$

where $n_{0}$ is the initial average number. In Fig. 2 we plot the expected mean phonon number over the first second of free evolution, starting from an initial number of $n_{0}=50$, showing the heating when the CSL mechanism is included (solid blue line), and when it is not (dashed orange line). In our case, the diffusive terms in Eq. (4) dominate over the dissipative, resulting in an expression that is approximately linear, as seen in Fig. 2. The inclusion of the CSL mechanism results in a heating rate of the nanosphere motion of about 2500 phonons/s, which is in stark contrast with the $\lambda_{\mathrm{csl}}=0$ case, where only $\sim 350$ phonons/s are achieved for the parameters used in our simulations.
Differentiating CSL from Conventional Noise.- If a final occupation number is recorded that agrees with the model of $\lambda_{\mathrm{csl}}=0$, we interpret it as falsifying a certain range of $\lambda_{\mathrm{csl}}$. However, if we measure a higher phonon number, we can infer that some extra heating process is present, possibly collapse effects. However, an objection could be made that the increased heating would more likely result from mis-characterising the environmental noise sources present.

Therefore, an important requirement is how to correctly identify CSL, and distinguish it from other noise sources. Indeed, this problem is generic to any test of collapse theories. We can address this problem by monitoring the conventional noise sources, such as blackbody radiation and gas collisions, by varying the associated parameters and determining the effect they have on the heating rate. For example, if the heating is strongly dominated by CSL, then varying the pressure will have little effect over some range, while a gas-collision dominated process results in an almost linear relation between pressure and heating rate.

Fig. 3 summarises such behaviours, and displays the trend followed by the mean phonon number after one second when varying different parameters for the cases of $\lambda_{\text {csl }}=10^{-8} \mathrm{~Hz}$ and for $\lambda_{\text {csl }}=0$. In Fig. 3 (a), we examine the effect of varying the background pressure when we include or exclude CSL. The difference in response between the theories is instructive. Without CSL the effect of an increasing pressure can be seen across the whole range, whereas for CSL there is a region of immunity where CSL dominates the dynamics. Likewise for the internal temperature depicted in Fig. 3 (b): the point at which this significantly influences the heating is different for the two theories. The response to a varying mechanical frequency also takes a different shape, as seen in Fig. 3 (c). Most interesting is the effect of a

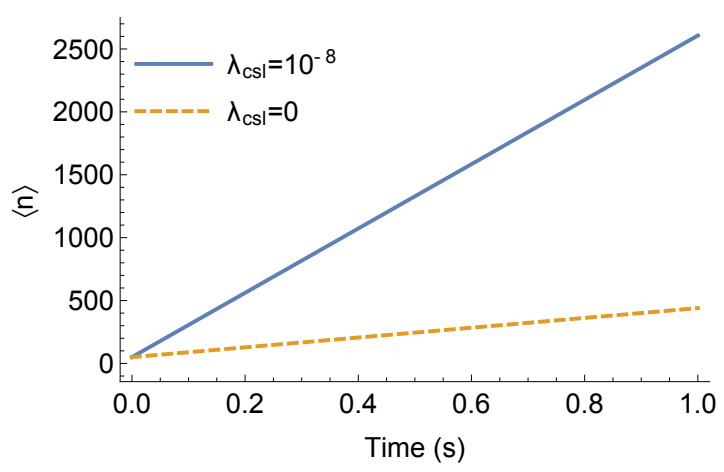

FIG. 2. (Color online) Expected phonon number $\langle n(t)\rangle$ over one second with and without collapse effects. We have used $n_{0}=50, R=100 \mathrm{~nm}$, a pressure of $10^{-12}$ mbar, $\omega_{m}=5 \mathrm{kHz}$, $\rho=2300 \mathrm{~kg} / \mathrm{m}^{3}, T_{\text {env }}=4 \mathrm{~K}$, and an internal temperature of $65 \mathrm{~K}$. The solid (dashed) line is for a CSL mechanism characterized by $\lambda_{\mathrm{csl}}=10^{-8},(0) \mathrm{Hz}$, i.e. the Adler value (no CSL mechanism). 
varying radius shown in Fig. 3 (d). Our findings agree with those of Ref. [11]: objects must be large enough to have an appreciable collapse rate, but small enough that decoherence does not dominate the dynamics in order for us to observe collapse effects. Such request is met for $R \in[10,100] \mathrm{nm}$, roughly. For all of these we have compared the Adler value for $\lambda_{\mathrm{csl}}=10^{-8} \mathrm{~Hz}$ with the case of no collapse effects. However the same differences persist for any chosen non-zero value of $\lambda_{\mathrm{csl}}$, though being more pronounced for higher collapse rates.

In order to find the best conditions for testing CSL it is necessary to numerically optimise all the parameters simultaneously. In Fig. 4, we show the range of $\lambda_{\text {csl }}$ which can be probed for an illustrative set of experimental conditions. We see that, as one would expect, the testable range depends ultimately upon the conditions that can be achieved, most relevantly the minimum value of both environmental pressure and internal temperature. The value of the initial phonon number and evolution time also play a significant role: after enough time, the phonon ratio will tend to the heating rate ratio as $\langle n(\infty)\rangle_{\mathrm{csl}} /\langle n(\infty)\rangle_{\mathrm{qm}} \rightarrow\langle\dot{n}\rangle_{\mathrm{csl}} /\langle\dot{n}\rangle_{\mathrm{qm}}$, where the denominator in each refers to the expected value when we take $\lambda_{\mathrm{csl}}=0$. The time required for the phonon ratio to approximate the heating ratio in this way depends upon the initial phonon number and heating rates. A lower initial phonon number or longer evolution times would promote each plot in Fig. 4, as a given set of parameters would be capable of probing a lower value of $\lambda_{\text {csl }}$.

Experimental Feasibility. - Charged silica particles of 200 $\mathrm{nm}$ have been trapped and cooled to milliKelvin temperatures in a Paul trap using cavity cooling to mil- (a)

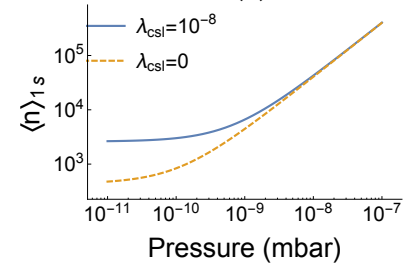

(c)

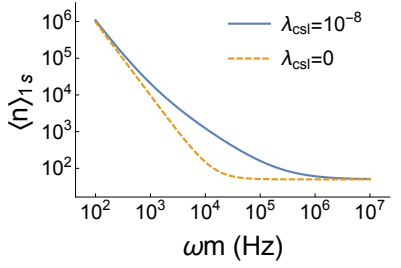

(b)

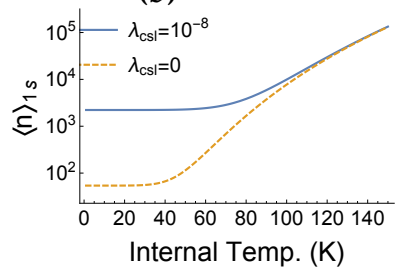

(d)

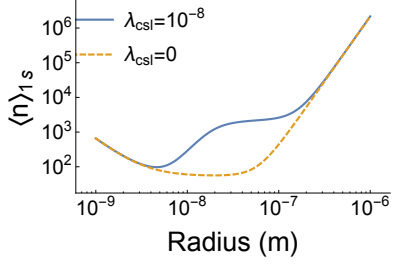

FIG. 3. (Color online) Phonon number after 1s as predicted by with (blue line) and without (dashed orange line) collapse effects as we vary the pressure in panel (a), bulk temperature in panel (b), mechanical frequency in panel (c), and the radius of the sphere in panel (d). Except where a parameter is under investigation, we have used the same values as in Fig. 2.

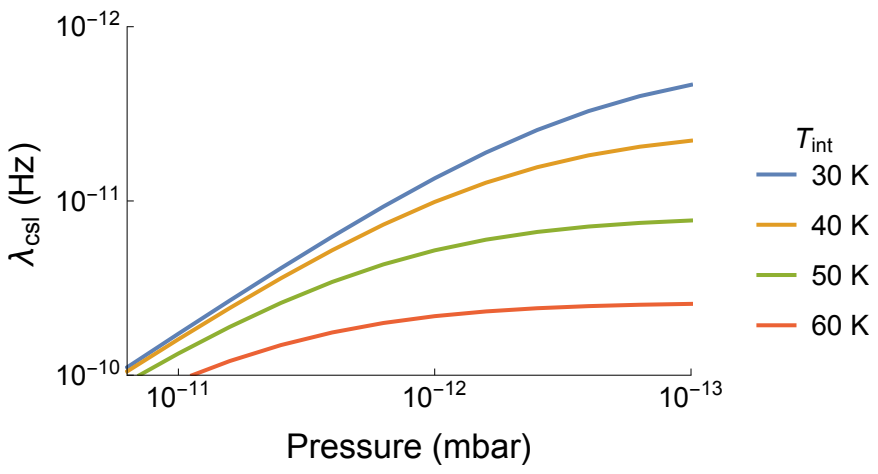

FIG. 4. (Color online) Each line shows the lowest value of $\lambda_{\mathrm{csl}}$ which could be tested for a given internal temperature $(30 \mathrm{~K}$, $40 \mathrm{~K}, 50 \mathrm{~K}, 60 \mathrm{~K}$ from top to bottom) as a function of environmental pressure. We take the lowest testable value to be the minimum for which $\langle n(100 \mathrm{~s})\rangle_{\mathrm{csl}} /\langle n(100 \mathrm{~s})\rangle_{\mathrm{qm}} \geq 1.2$ holds true, meaning that the we must expect the effect of CSL must cause a heating $20 \%$ greater than conventional noise alone over a period of 100 seconds, from a starting phonon number of $n_{0}=50$. The sphere radius and mechanical frequency have been optimised for each individual data point's temperature and pressure.

liKelvin temperatures [18] thus demonstrating the key experimental components required for our proposal. Pressures down to the $10^{-11} \mathrm{mbar}$ range and internal particle temperatures in the $10 \mathrm{~K}$ range can be obtained for this setup using standard cryopumps. An important component of these experiments is the measurement of the oscillator energy following parametric heating by CSL and conventional noise. These can be carried out by performing a homodyne measurement of position as a function of time which allows the determination of mean occupation number. Importantly, the light-cavity detuning can be non-perturbative during the measurement such that it neither cools nor heats the particle during this time. This ability to control the cooling rate also allows us to avoid giving strong kicks to the particle when we turn off the optical field after the particle is cooled to the desired energy.

Concluding Remarks.- We have shown that the parametric heating rate of a trapped nanosphere provides a viable mean of testing CSL. Central to the success of this scheme is the minimization of all sources of environmental decoherence. The two-stage 'cool and release' protocol that we have illustrated allows us to exploit optical cooling while avoiding problematic scattering noise that would otherwise dominate the dynamics. We remark that, owing to established techniques and common experimental settings, the experiment can be performed rapidly, and is repeatable upon a given nanosphere. Remarkably, the central experimental setup has already been demonstrated $[18,26]$. Our scheme can be used to distinguish CSL from other noise sources - an essential condition for inferring the existence of 
collapse effects from an experiment. Further, we have shown that the parameter range of CSL that is testable using our proposal is broad, and can readily be expected to probe $\lambda_{\text {csl }}=10^{10}\left(\lambda_{\text {csl }}=10^{-12}\right)$ using a background pressure of $10^{-11}$ mbar $\left(10^{-13} \mathrm{mbar}\right)$ and an internal temperature of $60 \mathrm{~K}(20 \mathrm{~K})$. Based on state of the art, values as low as $\lambda_{\mathrm{csl}}=10^{-8}$ could be tested imminently, and $\lambda_{\mathrm{csl}}=10^{-12}$ plausibly in the next few years.

Acknowledgements.-MP acknowledges the John Templeton Foundation (grant ID 43467), the UK EPSRC (EP/M003019/1), and the EU FP7 grant TherMiQ (Grant Agreement 618074) for financial support. DG and PFB also acknowledge the UK's EPSRC (EP/H050434/1, EP/J014664/1, EP/K026267/1), and thank $\mathrm{H}$. Ulbricht and M. Bahrami for useful discussions, and Jonathan Underwood for his help in modelling the rotational dynamics of the sphere.

* dangoldwater@gmail.com

[1] E. Joos, Decoherence and the appearance of a classical world in quantum theory (Springer, 2003).

[2] G. Ghirardi, P. Pearle and A. Rimini, Phys. Rev. A 42, 1 (1990).

[3] S. Adler, A. Bassi, S. Donadi, J. Phys. A: Math. Theor. 46 245304 (2013).

[4] Q. Fu, Phys. Rev. A, 563 (1997).

[5] A. Bassi, K. Lochan, S. Satin, T. P. Singh, and H. Ulbricht, Rev. Mod. Phys. 85, 471 (2013).

[6] W. Feldmann and R. Tumulka, J. Phys: Math. Theor. 45 065304 (2012).

[7] S. L. Adler, J. Phys. A 402935 (2006).

[8] F. Laloe, W. Mullin, P. Pearle, Phys. Rev. A 90052119 (2014).

[9] A. Bassi, D.-A. Deckert, and L. Ferialdi, Europhys. Lett. 92, 50006 (2010).

[10] A. Bassi and G. Ghirardi, Phys. Rep. 379, 257 (2003).

[11] S. Nimmrichter, K. Hornberger, and K. Hammerer, Phys. Rev. Lett. 113, 020405 (2014).

[12] P. Sekatski, M. Aspelmeyer, and N. Sangouard, Phys. Rev. Lett. 112, 080502 (2014).

[13] O. Romero-Isart, Phys. Rev. A 84, 052121 (2011).

[14] C. Emary, J. P. Cotter, and M. Arndt, Phys. Rev. A 90, 042114 (2014).

[15] M. Arndt and K. Hornberger, Nat. Phys. 10, 271 (2014).

[16] J. Bateman, S. Nimmrichter, K. Hornberger, and H. Ulbricht, Nat. Commun. 5, 4799 (2014).
[17] See Supplementary Information accompanying the paper, available at XXX.

[18] P. Z. G. Fonseca, E. B. Aranas, J. Millen, T. S. Monteiro, P. F. Barker, arXiv:1511.08482 (2015).

[19] M. Bahrami, M. Paternostro, A. Bassi, and H. Ulbricht, Phys. Rev. Lett. 112, 210404 (2014).

[20] C. Arenz, R. Hillier, M. Fraas, and D. Burgarth, arXiv:quant-ph/1405.7644 (2014).

[21] S. L. Adler, J. Phys. A 38, 2729 (2005).

[22] B. Collette, P. Pearle, Found. Phys., 3310 (2003).

[23] S. Bera, B. Motwani, T. P. Singh, and H. Ulbricht, Sci. Rep. 5, 7664 (2015).

[24] L. Diosi, Phys. Rev. Lett. 114, 050403 (2015).

[25] C. Gardiner and P. Zoller, Quantum noise (Springer, 2004).

[26] J. Millen, P. Z. G. Fonseca, T. Mavrogordatos, T. S. Monteiro, and P. Barker, Phys. Rev. Lett. 114, 123602 (2015).

[27] I. Wilson-Rae, N. Nooshi, W. Zwerger, and T. Kippenberg, Phys. Rev. Lett. 99, 093901 (2007).

[28] J. Gieseler, B. Deutsch, R. Quidant, and L. Novotny, Phys. Rev. Lett. 109, 103603 (2012).

[29] P. F. Barker, J. Millen, Y. Lia Li, M. Trivedi, and T. S. Monteiro, SPIE NanoScience+ Engineering, 845808 (2012).

[30] C. Genes, D. Vitali, P. Tombesi, S. Gigan, and M. Aspelmeyer, Phys. Rev. A, 77, 033804 (2008).

[31] P. Barker and M. N. Shneider, Phys. Rev. A 81, 023826 (2010).

[32] D. E. Chang, C. a. Regal, S. B. Papp, D. J. Wilson, J. Ye, O. J. Painter, H. J. Kimble, and P. Zoller, Proc. Nat. Acad. Sci. USA 107, 1005 (2010).

[33] O. Romero-Isart, M. L. Juan, R. Quidant, and J. I. Cirac, New J. Phys. 12, 033015 (2010).

[34] A. C. Pflanzer, O. Romero-Isart, and J. I. Cirac, Phys. Rev. A 86, 013802 (2012).

[35] O. Romero-Isart, A. C. Pflanzer, M. L. Juan, R. Quidant, N. Kiesel, M. Aspelmeyer, and J. I. Cirac, Phys. Rev. A 83, 013803 (2011).

[36] T. S. Monteiro, J. Millen, B. Pepper, F. Marquardt, D. E. Chang, and P. Barker, New J. Phys. 15, 015001 (2013).

[37] M. Paternostro, S. Gigan, M. S. Kim, F. Blaser, H. R. Böhm, and M. Aspelmeyer, New J. Phys. 8, 107 (2006).

[38] In order to conserve energy, CSL must include a dissipative mechanism that would compensate for the energy imparted by random collapses. A. Smirne and A. Bassi, arXiv:quant-ph/1408.6446, have developed a model for such mechanism. As we show in the Supplementary Information, the effect of this mechanism upon our system is negligible.

[39] F. Marquardt, J. Chen, A. A. Clerk, and S. Girvin, Phys. Rev. Lett. 99, 093902 (2007).

[40] S. L. Adler and A. Bassi, J. Phys. A 40, 15083 (2007).

[41] B. Rodenburg, L. P. Neukirch, A. N. Vamivakas, M. Bhattacharya, arXiv:1503.05233 (2015). 